\title{
Real-Time Pleural Elastography: Potential Usefulness in Nonintubated Video-Assisted Thoracic Surgery
}

\author{
Federico Tacconi, M.D. ', Fabrizio Chegai, M.D.', Tommaso Perretta, M.D., Ph.D.', Vincenzo Ambrogi, M.D., Ph.D. ${ }^{1}$ \\ 'Unit of Thoracic Surgery and 'Department of Diagnostic Imaging, Interventional Radiology, Molecular Imaging and Radiotherapy, Tor Vergata University \\ Hospital, Rome, Italy
}

\section{ARTICLE INFO}

Received September 23, 2020

Revised October 22, 2020

Accepted November 3, 2020

Corresponding author

Federico Tacconi

Tel 39-06-20902883

Fax 39-06-20902894

E-mail Federico.Tacconi@ptvonline.it ORCID

https://orcid.org/0000-0001-8850-5027
Pleural adhesions are a major challenge in standard and nonintubated video-assisted thoracic surgery. The currently available imaging techniques help to assess the presence and extent of pleural adhesions, but do not provide information on tissue deformability, which is crucial for intraoperative management. In this report, we describe the utilization of real-time elastography mapping of pleural adhesions. This technique enabled us to detect areas with softer adhesions, and helped establish the surgical plan in a difficult case of a patient scheduled for nonintubated video-assisted thoracic surgery.

Keywords: Ultrasound, Video-assisted thoracic surgery, Pleura

\section{Introduction}

Pleural adhesions (PAs) are among the main determinants of the feasibility and time-efficiency of video-assisted thoracic surgery (VATS). This is particularly true in nonintubated VATS, as PAs represent the most frequent cause of conversion to general anesthesia and thoracotomy [1]. Chest ultrasonography may be useful to predict PAs [2], with sensitivity ranging from $50 \%$ to $80 \%[3,4]$. However, chest ultrasonography alone does not provide information on PA elasticity, which is crucial for intraoperative management. Real-time tissue elastography (RTE) ultrasonographically assesses tissue deformability under mechanical stress. In this report, RTE was used for this purpose and helped to establish the surgical plan.

\section{Technique}

We used RTE for the very first time in a 67-year-old patient with a past medical history of multiple solid tumors and recent onset of bilateral lung nodules. After an uninformative imaging-guided needle biopsy, the multidisciplinary team meeting made the decision to offer surgical biopsy. The patient had also received bypass surgery 3 years earlier. In an interview, he reported having had a pleural effusion in his 40s (side unknown). Chest computed tomography (CT) showed no relevant pleural thickening bilaterally (Fig. 1A, B). The patient consented to undergo nonintubated VATS biopsy, after a thorough discussion of the expected benefits and potential risk of the offered plan. Given that chest ultrasonography was deemed to be a part of usual clinical practice with virtually no risk to the patient, the Institutional Review Board waived the need for formal approval of the technique. The patient provided written informed consent for the publication of his clinical details and images.

Real-time elastography images were obtained by gentle probe compression on the anterior, lateral, and posterior aspects of the chest at 3 different intercostal spaces per side, with a total 18 regions of interest. An L5-12 linear probe connected to an iU22 ultrasound machine (Philips Healthcare, Eindhoven, the Netherlands) was used. The elastographic images were generated by a dedicated algorithm (Elasto; Hitachi Medical Systems, Tokyo, Japan) and displayed alongside the standard grayscale B-mode ultrasound images. Red areas indicated soft, deformable tissue, whereas green areas indicated different degrees of stiffness (Fig. 1C-F). The thoracic surgeons involved in the case were present for the RTE examination.

The RTE mapping showed a prevailing pattern of homo- 

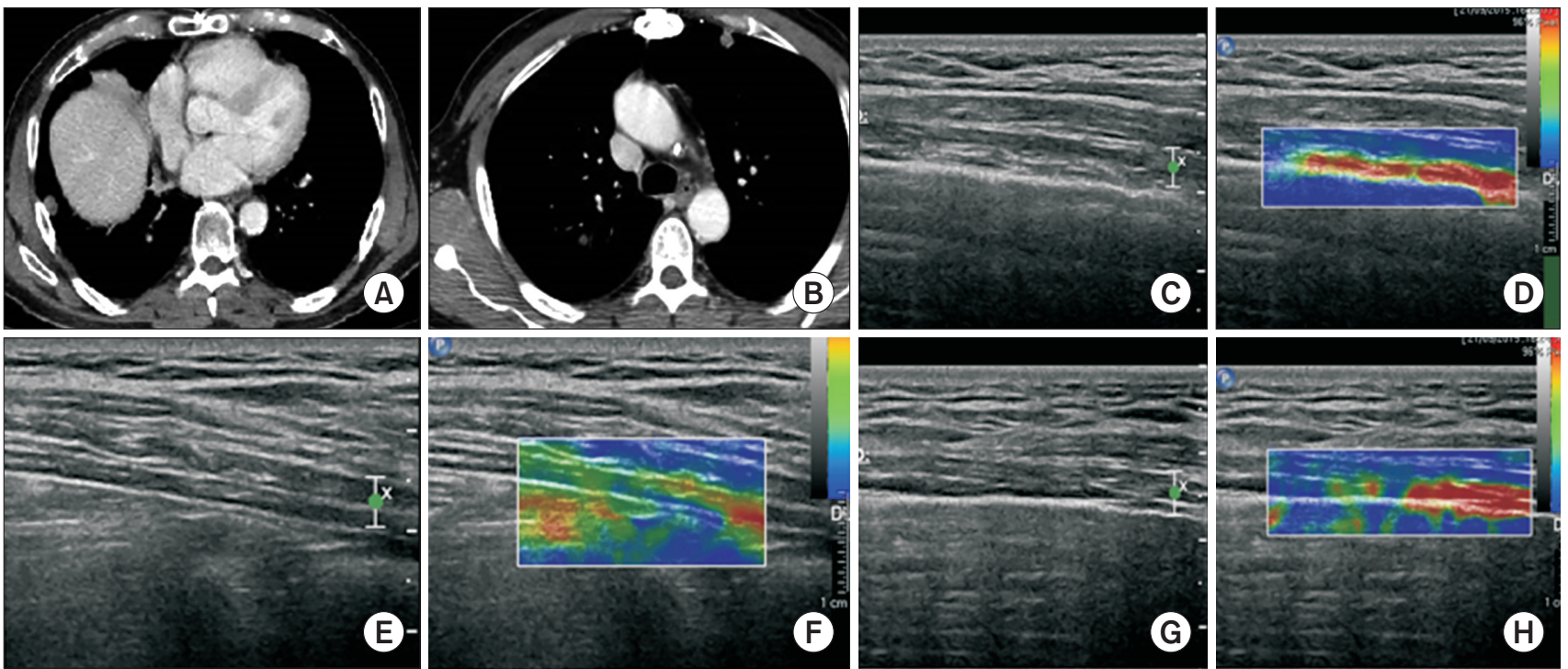

Fig. 1. (A, B) Computed tomography scan showing bilateral lung nodules and no pleural thickening. (C, D) Homogeneous, soft adhesions, $(\mathrm{E}, \mathrm{F})$ stiff tissue deposits, and $(\mathrm{G}, \mathrm{H})$ a mosaic-like pattern with mixed features found with real-time elastography in the same patient.

geneous, soft PAs on the right side, surrounded by scant spots of stiffer tissue (Fig. 1C, D) in 6 of the 9 regions of interest assessed. On the left side, there were abundant stiff tissue deposits (green areas, Fig. 1E, F; 4 regions of interest). Both sides had some areas with a mosaic-like pattern, consisting of thick tissue islets alternating with soft adhesions (Fig. 1G, H).

During right uniportal nonintubated VATS, we found abundant soft and elastic adhesions, alternating with sparse areas of dense, vascularized adhesions (Fig. 2). This feature was in keeping with the RTE findings, and consistent with type II/III PA according to the classification proposed by Li et al. [5]. The patient's postoperative recovery was uneventful and he was discharged on postoperative day 2.

\section{Discussion}

PAs are a highly relevant determinant of postoperative outcomes in VATS. In the specific setting of nonintubated operations, PA can jeopardize feasibility and require conversion to general anesthesia, which is a less than ideal scenario in terms of safety and time-efficiency. Furthermore, conversion can be perceived as a treatment failure by patients and their relatives. Therefore, preoperative detection of PAs can be useful when the risk-to-benefit ratio of attempting a suboptimal nonintubated operation should be balanced against the advantage of upfront standard VATS with general anesthesia.

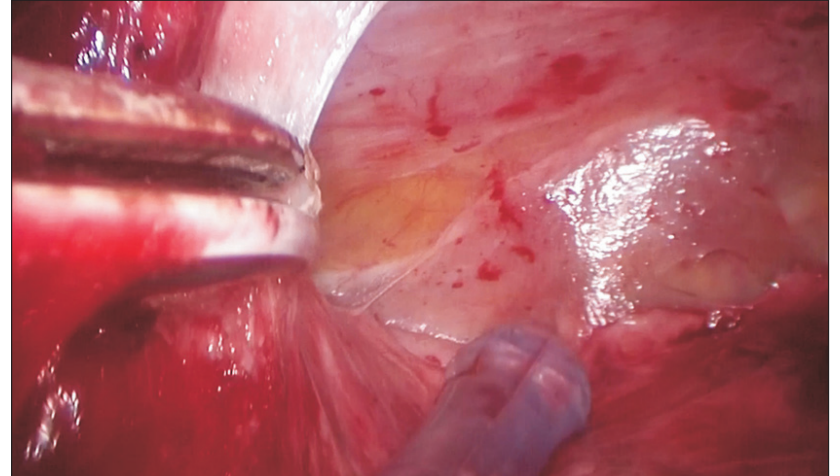

Fig. 2. Intraoperative picture of the same patient, showing elastic and poorly vascularized adhesions that are easily retracted (right), alongside with slightly stiffer tissue (left).

Real-time elastography has been widely used in orthopedics, mainly for assessing tendon diseases. In pulmonology, it has been occasionally used as a tool to distinguish pleural empyema from transudate, and for the assessment of subpleural and pulmonary lesions [6]. To our knowledge, no previous reports have described RTE as a method for assessing PA preoperatively. In this single case, RTE helped us plan the operation by showing homogeneously distributed soft adhesions in the right hemithorax, compared to remarkably stiffer adhesions in the left. Based on our experience, we reasoned that preoperative RTE mapping of PAs may result in other advantages whenever the presence of PAs may be a concern. Indeed, the use of RTE mapping may help the patient become more clearly aware of possible 
pitfalls of surgery. It might also be useful in fine-tuning the selection of optimal candidates during the initial training phase of a nonintubated VATS program. Furthermore, it might provide stronger evidence in cases of legal concern following surgical complications.

A limitation of RTE is that some chest areas, such as the scapular and apical regions, are not accessible by the ultrasound probe. In this regard, we suggest that the main goal of RTE is to rule out thick adhesions in the majority of the explored regions. If this is the case, it is likely that the lung may be still retracted easily, thereby making nonintubated VATS feasible. Yet another limitation is that in our preliminary experience, RTE is unfeasible in overweight patients.

In conclusion, this clinical case demonstrates the potential of RTE for optimizing surgical planning in nonintubated VATS operations. Further larger-scale investigations are warranted to assess the validity and usefulness of RTE in daily practice.

\section{Conflict of interest}

No potential conflict of interest relevant to this article was reported.

\section{ORCID}

Federico Tacconi: https://orcid.org/0000-0001-8850-5027

Fabrizio Chegai: https://orcid.org/0000-0001-8736-6475

Tommaso Perretta: https://orcid.org/0000-0002-5282-4357

Vincenzo Ambrogi: https://orcid.org/0000-0002-8947-3175

\section{References}

1. Mineo TC, Tacconi F. From "awake" to "monitored anesthesia care" thoracic surgery: a 15 year evolution. Thorac Cancer 2014;5:1-13.

2. Jeong JY, Park HJ, Shin JS, Jo WM, Lee IS. Ultrasound for detecting pleural adhesion before video-assisted thoracic surgery. Korean J Thorac Cardiovasc Surg 2010;43:399-403.

3. Cassanelli N, Caroli G, Dolci G, et al. Accuracy of transthoracic ultrasound for the detection of pleural adhesions. Eur J Cardiothorac Surg 2012;42:813-8.

4. Jeong H, Choi JW, Ahn HJ, Choi J, Park JH. Prediction of pleural adhesions by lung ultrasonography: an observational study. J Cardiothorac Vasc Anesth 2020:S1053-0770(20)30571-1.

5. Li SJ, Zhou K, Wu YM, et al. Presence of pleural adhesions can predict conversion to thoracotomy and postoperative surgical complications in patients undergoing video-assisted thoracoscopic lung cancer lobectomy. J Thorac Dis 2018;10:416-31.

6. Sperandeo M, Trovato FM, Dimitri L, et al. Lung transthoracic ultrasound elastography imaging and guided biopsies of subpleural cancer: a preliminary report. Acta Radiol 2015;56:798-805. 